\title{
Spectral Detection of an Intermediate Preceding the Excited State in the Bacterial Luciferase Reaction ${ }^{\dagger}$
}

\author{
Peter Macheroux, ${ }^{\ddagger}, 8$ Sandro Ghisla ${ }^{\ddagger}$ and J. Woodland Hastings ${ }^{*, 8}$ \\ Faculty of Biology, University of Konstanz, P.O. Box 5560, D-78434 Konstanz, Germany, and Biological Laboratories, Harvard \\ University, Cambridge, Massachusetts 02138-2020
}

\begin{abstract}
The bioluminescent reaction of luciferase isolated from Vibrio harveyi, strain M17, was initiated by mixing the luciferase-bound flavin 4a-hydroperoxide intermediate, purified in advance, with a long-chain aldehyde (dodecanal or octanal) at $-4{ }^{\circ} \mathrm{C}$. Measurements of absorbance changes from 300 to $600 \mathrm{~nm}$ during the course of the reaction revealed the existence of three sets of isosbestic points and three kinetic phases, the second of which parallels kinetically the decay of bioluminescence, measured concurrently. The absorbance changes in this second step and the decay of light emission exhibited similar deuterium isotope effects; this is postulated to be the step giving rise to the excited state and the enzyme-bound flavin 4ahydroxide. The first step of the reaction, however, did not show an isotope effect; the intermediate thereby formed, observed here for the first time, is postulated to correspond to the luciferase-bound flavin 4aperoxyhemiacetal.
\end{abstract}

Of the many light-emitting organisms, only the bacteria produce a luciferase that utilizes a flavin as a substrate and the emitter (Hastings, 1983). Bacterial luciferase (EC 1.14.14.3) is a monooxygenase which gives rise to light emission upon reaction of enzyme-bound $\mathrm{FMNH}_{2}{ }^{1}$ with molecular oxygen and a long-chain aldehyde. The products are oxidized FMN, the corresponding fatty acid, and water (Hastings et al., 1985; Baldwin \& Ziegler, 1991). The postulated pathway and principal intermediates (all luciferase-bound) are shown in Scheme 1.

A key intermediate (II), which is formed by reaction of $\mathrm{FMNH}_{2}$ with oxygen and occurs prior to reaction with aldehyde, is the flavin 4a-hydroperoxide (Hastings et al., 1973; Vervoort et al., 1986). An intermediate formed concurrently with light emission (IV) was isolated and characterized by Kurfürst et al. (1984, 1987). However, an intermediate (III) postulated to occur upon reaction of II with aldehyde via the Michaelis complex (IIA), but preceding light emission, remained to be demonstrated.

Since Kurfürst et al. (1984) used decanal, a fast-reacting aldehyde, we thought it possible that the existence of this earlier intermediate might be easier to detect under conditions where the reaction proceeds more slowly. Accordingly, dodecanal and octanal were selected as aldehydes (Hastings et al., 1969), and experiments were performed at a lower temperature $\left(-4^{\circ} \mathrm{C}\right)$.

The reactions were carried out starting with the luciferasebound flavin 4a-hydroperoxide (II) prepared and purified in advance. Concurrent measurements of absorption and light emission were made. The results show three sets of isosbestic points, indicating the existence of an additional intermediate, which is postulated to correspond to the luciferase flavin 4a-

† This work was supported by grants to J.W.H. from the U.S. Office of Naval Research (N00014-92-J-1657) and the U.S. National Science Foundation (MCB-9306879) and from the Deutsche Forschungsgemeinschaft to S.G. (DFG Gh 2/4-5,6).

$\$$ University of Konstanz.

Harvard Univeristy.

- Abstract published in Advance ACS Abstracts, December 1, 1993.

${ }^{1}$ Abbreviations: FMN and FMNH ${ }_{2}$, riboflavin 5'-phosphate and its reduced form; NMR, nuclear magnetic resonance. peroxyhemiacetal (III).

\section{EXPERIMENTAL PROCEDURES}

Cells of Vibrio harveyi strain M17 (Ulitzur \& Hastings, 1978) were grown and harvested as described by Nealson (1978). Luciferase was extracted and purified according to the procedure of Holzman and Baldwin (1982). The enzyme purity was estimated by gel electrophoresis to be $>95 \%$. Luciferase concentration was determined using $M_{\mathrm{r}}=79000$ and an absorption coefficient of $1.2(0.1 \%, 1 \mathrm{~cm})$ at $280 \mathrm{~nm}$ (Gunsalus-Miguel et al., 1972). Preparations (about $1 \mathrm{~mL}$ ) of the luciferase flavin 4a-hydroperoxide $(8-12 \mu \mathrm{M})$ were obtained by the procedure of Kurfürst et al. (1984). Ethylene glycol was added to buffers to a final concentration of $10 \%$. In addition to helping solubilize aldehyde, ethylene glycol acts as an antifreeze. It does not inhibit bioluminescence in the concentration used; indeed, it stimulates slightly (Hastings \& Balny, 1975).

FMN was purchased from Sigma (grade I, F2253) and was not purified further. Octanal and dodecanal were obtained from Aldrich, distilled prior to use, and dissolved in ethylene glycol, giving nonturbid solutions of known concentrations. The $C(1)$-deuteriated aldehydes were synthesized following the procedure described by Seebach et al. (1966). Purities of the aldehydes were determined by gas chromatography to be $>98 \%$ and the isotopic purity was $>95 \%$ as judged by ${ }^{1} \mathrm{H}$ NMR spectroscopy.

A commercial spectrophotometer (Kontron, Uvikon 820) was equipped to measure the kinetics of both absorbance and light emission at temperatures as low as $-4^{\circ} \mathrm{C}$ (Kurfürst et al., 1984). Reactions were initiated in the cuvette by mixing $900 \mu \mathrm{L}$ of the luciferase-bound FMN 4a-hydroperoxide (in $10 \mathrm{mM}$ potassium phosphate and $350 \mathrm{mM}$ sodium chloride buffer, $\mathrm{pH}$ 7) with $100 \mu \mathrm{L}$ of the aldehyde solution.

\section{RESULTS}

Panels A, B, and C of Figure 1 show spectra recorded during the course of the reaction of purified luciferase-bound flavin 
Scheme 1: Sequence of Steps and Intermediates Proposed To Reflect the Observed Spectral Changes ${ }^{a}$

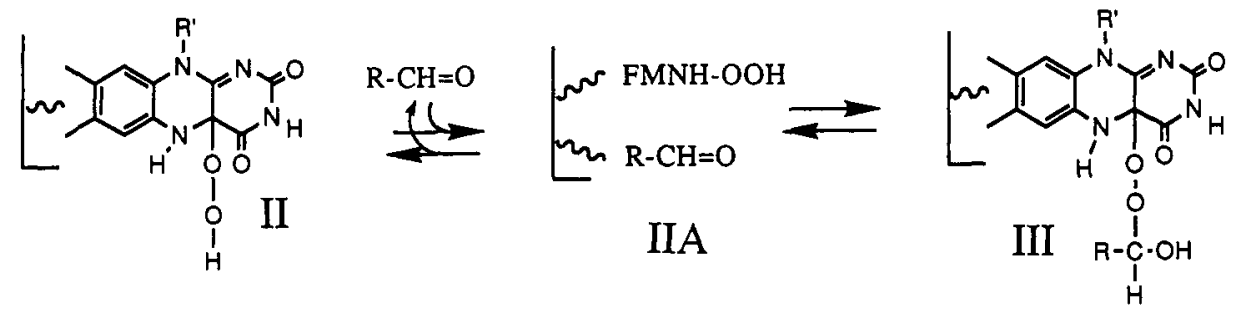

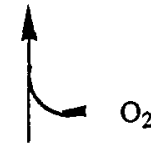

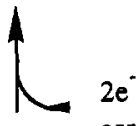
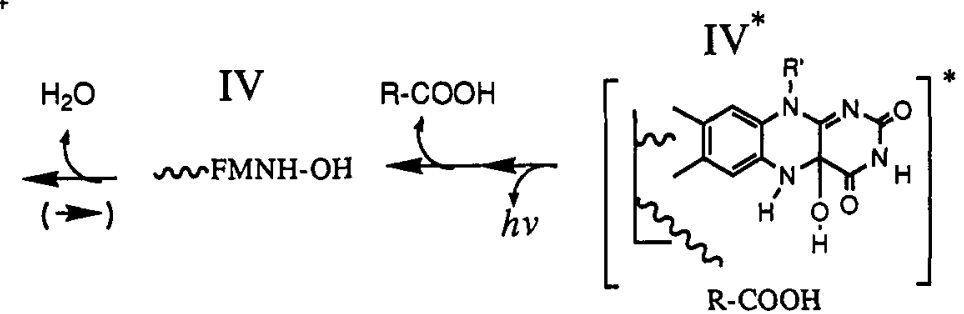

' FMN, flavin mononucleotide; $\mathbf{R}^{\prime}$, ribityl phosphate; $\mathbf{R}$, dodecyl or heptyl.

4a-hydroperoxide (II) with dodecanal deuteriated at position $\mathrm{C}(1)$. At $-4^{\circ} \mathrm{C}$ the reaction is slow enough to allow absorbance and photometric measurements using a conventional spectrophotometer equipped for simultaneous recording of both. In panel $A$, curve 0 depicts the absorption spectrum of the purified flavin 4a-hydroperoxide (II); curve 1 was recorded immediately after the addition of aldehyde and is displaced from curve 0 by virtue of dilution and the increased turbidity due to the aldehyde. Curves 2-5 were obtained sequentially in time up to $12.5 \mathrm{~min}$ after the addition of dodecanal and show the first set of isosbestic points at 326,378 , and $398 \mathrm{~nm}$.

In panel B, curves 6-10 were recorded every 8 min between 15 and $47 \mathrm{~min}$ and reveal a second set of isosbestic points $(335,384$, and $408 \mathrm{~nm})$. Panel $\mathrm{C}$ shows the terminal phase of the reaction; traces 11-15 were recorded at intervals between 51 and $111 \mathrm{~min}$, and isosbestic points at 344, 382, and 412 nm were observed during this phase. The final spectrum shown (trace 16) was taken subsequent to warming to $20^{\circ} \mathrm{C}$ and recooling to $-4{ }^{\circ} \mathrm{C}$, after which no further spectral changes occurred.

Many similar measurements were carried out with protiododecanal and also with protio- and deuteriooctanal; these gave similar spectral changes with time and similar isosbestic points. Table 1 summarizes the results of 10 such experiments, where the recordings were of a quality similar to the one shown in Figure 1. One difficulty, as mentioned above, was the turbidity introduced by the addition of aldehyde; however, it usually remained constant over the course of the recordings and so did not obscure the isosbestic points. However, as in any reaction with more than two species, the isosbestic points may be inexact because of the absorbance of earlier or later reaction components. This must certainly be assumed to be so in the present experiments.

However, the occurrence of three sets of apparent isosbestic points indicates that three phases of the reaction can be distinguished. Thus there are (at least) two spectrally observable intermediates between the luciferase-bound flavin $4 a$-hydroperoxide (II) and the end product, oxidized FMN
(V), and the kinetics of the steps may be determined by absorbance changes at appropriate wavelengths.

The first step was evaluated by measurements at one of the second isosbestic points $(408 \mathrm{~nm})$. The changes were relatively rapid, with a half-time of about $150 \mathrm{~s}$ (Figure 2, top panel, triangles). When the same measurements were made using 1-protiododecanal (Figure 2, bottom panel), a half-time of $200 \mathrm{~s}$ was obtained. On the basis of several such determinations, there was no significant difference between the two (Table 1). From this it may be deduced that the spectral changes occurring in this first phase precede the step(s) involving breaking of the aldehyde $\mathrm{C}(1)-\mathrm{H}$ bond, which is (are) postulated to occur during formation of IV* (Scheme 1).

The second phase was monitored by spectral changes occurring at a first-phase isosbestic point $(378 \mathrm{~nm})$, where there is a clear absorbance change during this time (Figure 1 , panel B). The absorbance change had a half-time of about $990 \mathrm{~s}$ (Figure 2, top panel, diamonds). With the protioaldehyde, the half-time was about $640 \mathrm{~s}$, yielding an isotope effect of $\sim 1.5$ (Table 1 ).

The rate of this second step corresponds closely to the rate of the decay of the luminescence (Figure 2, solid circles) and may thus be identified with the step involving breaking the aldehyde $\mathrm{C}(1)$ bond and formation of the excited state IV*, whose lifetime is assumed to be much shorter ( $\sim 1 \mathrm{~ns})$. The expected product, then, is the ground state of this species, the luciferase-bound flavin 4a-hydroxide (IV), previously identified by Kurfürst et al. $(1984,1987)$. A fluorescent transient described by Matheson and Lee (1983) is most likely the same species.

There is a final (third) phase, which is significantly slower than the decay of light emission and which leads to the final increase of absorbance at $440 \mathrm{~nm}$ (Figure 2, stars). It reflects the loss of water from intermediate (IV) to form oxidized FMN (V) (Kurfürst et al., 1984), but with dodecanal it also has an isotope effect of about 1.6. Since elimination of $\mathrm{H}_{2} \mathrm{O}$ itself is not expected to have a deuterium isotope effect, and 

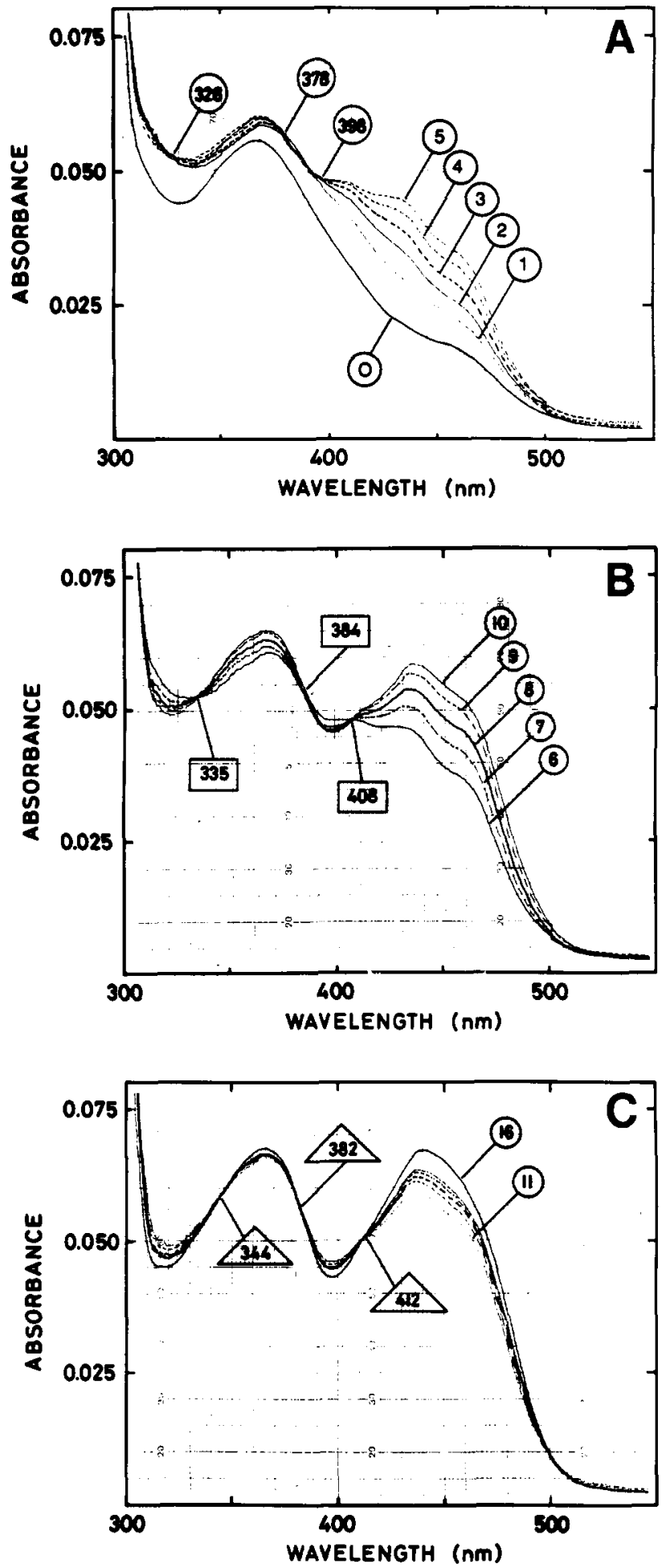

FIGURE 1: Spectral changes associated with reaction of the luciferase flavin 4a-hydroperoxide with $\mathrm{C}(1)$ deuteriododecanal in $10 \mathrm{mM}$ potassium phosphate and $350 \mathrm{mM}$ sodium chloride buffer, $\mathrm{pH}$ 7, at $-4^{\circ} \mathrm{C}$. (A) Absorbance spectrum (0) represents the starting spectrum of freshly purified luciferase flavin 4a-hydroperoxide $(900 \mu \mathrm{L})$. Dodecanal $(100 \mu \mathrm{L})$ was mixed with the peroxide and after $30 \mathrm{~s}$ spectrum 1 was recorded. The subsequent curves $(2-5)$ were recorded after 3.5, 6.5, 9.5, and $12.5 \mathrm{~min}$, respectively. The isosbestic points are noted. An increase in absorbance occurs at all wavelengths upon aldehyde addition due to scattering. (B) The same reaction with spectral changes represented by the absorbance spectra 6-10, recorded at $15,23,31,39$, and $47 \mathrm{~min}$, respectively. The isosbestic points are noted. (C) In the final phase, spectra were recorded at $51,66,81$, 96 , and $111 \mathrm{~min}$. Trace 16 represents the final spectrum recorded after warming the sample to $25^{\circ} \mathrm{C}$ for $10 \mathrm{~min}$ (after this time no further spectral changes could be detected) and subsequent recooling to $-4{ }^{\circ} \mathrm{C}$. indeed with octanal it does not, the observed slower rate found with deuteriated dodecanal might be due to the preceding step, as would be expected when the two relevant rate constants do not differ by a large amount (Table 1).

\section{DISCUSSION}

It may be remarked that the measurements of spectral changes in these experiments are based on very small absolute absorbance values and that sequential reactions and several components are involved. It is thus perhaps surprising that the isosbestic points and the three phases can be distinguished so well. However, the experiments were repeated many times with similar results, with both octanal and dodecanal (Table 1). Small differences in the rates and in the positions of the isosbestic points, for a given aldehyde, are attributed to experimental error.

The results obtained can be interpreted on the basis of the steps and intermediates proposed earlier (Kurfürst et al., 1984), shown in Scheme 1. Recent kinetic studies (Abu-Soud et al., 1992) confirmed this basic scheme; although their measurements involved the overall bacterial luciferase reaction, they assigned rate constants to many of the individual steps. Their study differed from ours in many ways and thus, except for the same reaction scheme, is not readily compared. They initiated reactions with luciferase, $\mathrm{FMNH}_{2}$, and oxygen at 25 ${ }^{\circ} \mathrm{C}$, while we studied the reaction at $-4{ }^{\circ} \mathrm{C}$ starting with the intermediate luciferase-bound flavin 4a-hydroperoxide (II), purified in advance. They utilized decanal whereas we used octanal and dodecanal; this will have a large effect on the kinetics of the individual steps. In spite of these differences, the phases and rate constants reported here are in good agreement with the scheme of Abu-Soud et al. (1992). In the present experiments the resolution of the different phases further allowed us to localize a deuterium isotope effect.

The observed spectral changes in phase 1 cannot be interpreted simply in terms of the luciferase-bound flavin 4ahydroperoxide complex with aldehyde, since spectral changes associated with that are essentially complete within $1 \mathrm{~min}$ at a much lower temperature $\left(-30^{\circ} \mathrm{C}\right)$ and in any event are much smaller (Hastings \& Balny, 1975). We propose that these changes, i.e., phase 1 , are associated with the formation of an intermediate resulting from the reaction of the peroxide with aldehyde, previously postulated to be the luciferase-bound flavin 4a-peroxyhemiacetal (III) (Eberhard \& Hastings, 1972). The formation of a ternary aldehyde $C(1)$ peroxy adduct is chemically reasonable and consistent with the observation that the luciferase reaction carried out with ${ }^{18} \mathrm{O}_{2}$ leads to the incorporation of one atom of ${ }^{18} \mathrm{O}$ into the fatty acid and the other into a water molecule (Suzuki et al., 1983). This intermediate, whatever its structure may be, has not been previously observed.

The initial rise of luminescence emission is fast, certainly as rapid as the spectral changes attributed to the formation of the first intermediate. In the experiment of Figure 1, for example, the half-time for the rise of luminescence was measured as $70 \mathrm{~s}$, compared with $150 \mathrm{~s}$ for the absorption changes at $408 \mathrm{~nm}$, corresponding to the first phase. This rapid onset of bioluminescence is considered to be spurious and is attributed to the biphasic character of the reaction; there is an initial faster phase, previously shown by rapid mixing and recording techniques (Presswood \& Hastings, 1979), which cannot be resolved in the measurements made here. As can be seen in Figure 2, top panel, the peak of luminescence is not reached until the first phase is nearly complete, i.e., after most of II has been converted to III. The 


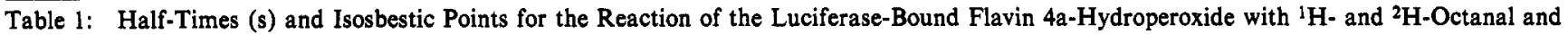
${ }^{1} \mathrm{H}$ - and ${ }^{2} \mathrm{H}$-Dodecanal

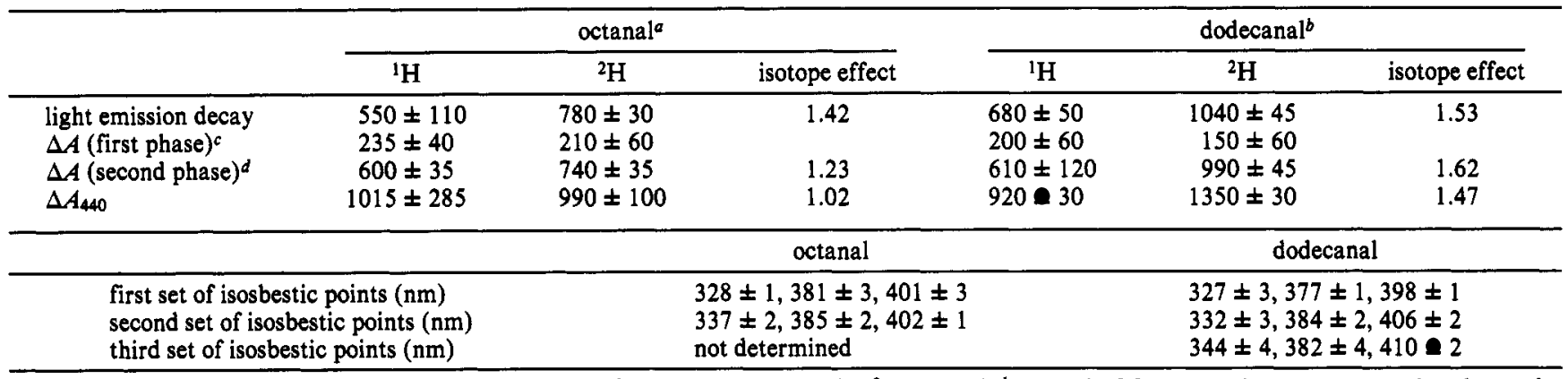

${ }^{a}$ A total of six experiments were analyzed, four with ${ }^{1} \mathrm{H}$-octanal and two for ${ }^{2} \mathrm{H}$-octanal. ${ }^{b} \mathrm{~A}$ total of four experiments were analyzed, two for ${ }^{1} \mathrm{H}$-dodecanal and two for ${ }^{2} \mathrm{H}$-dodecanal. ${ }^{c}$ Half-times for the first phase were determined at one of the isosbestic points of the second set. ${ }^{d} \mathrm{Half}$-times for the second phase were determined at one of the isosbestic points of the first set.
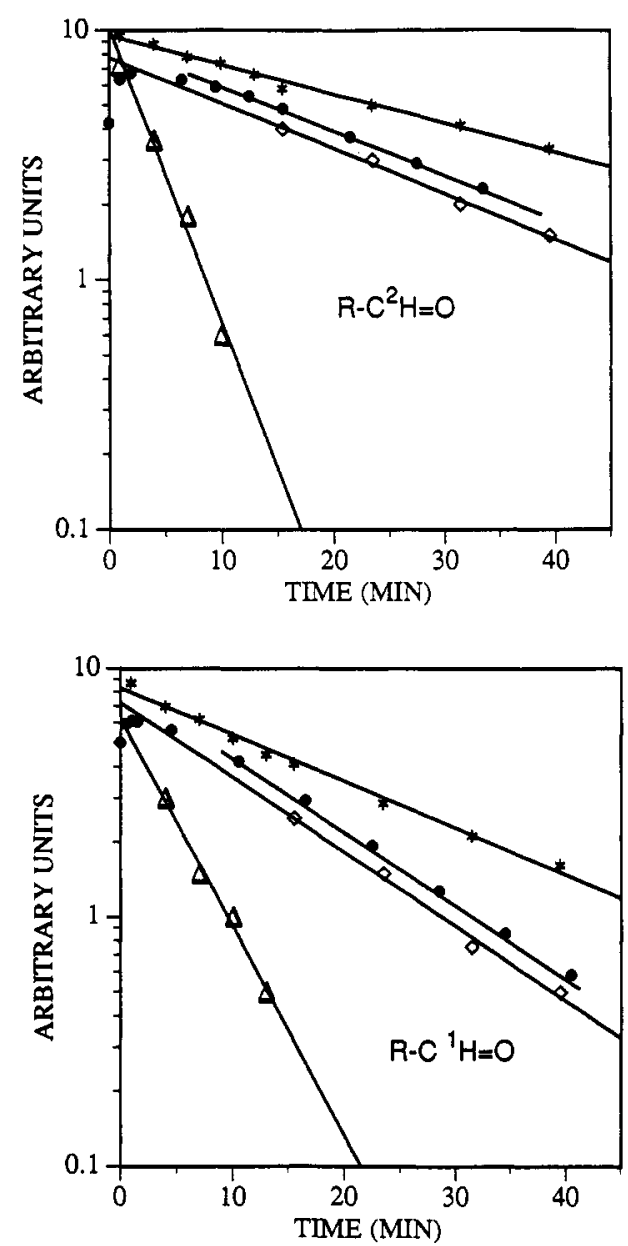

FIGURE 2: Semilogarithmic plots of the spectral and bioluminescence emission changes occurring with time during the reaction of purified flavin 4a-hydroperoxide with deuterio- and protiododecanal. (Top panel) Deuteriododecanal. The symbols refer to spectral changes at $408 \mathrm{~nm}$ (triangles), $378 \mathrm{~nm}$ (diamonds), and $440 \mathrm{~nm}$ (stars). The time course of bioluminescence emission is shown by the solid circles. (Bottom panel) Protiododecanal. Symbols refer to the same measurements as in the top panel.

peak of luminescence and the maximum in the amount of III are thus estimated to occur at the same time, and their decay thereafter occurs in parallel (Figure 2).

The fact that similar isotope effects were observed for the decay of bioluminescence emission intensity and the spectral changes in the second phase further indicates that this step reflects the formation of the excited state of the emitter and thus provides additional support for the conclusion of Kurfürst et al. $(1984,1987)$ that the ground state of the emitter is the flavin $4 \mathrm{a}$-hydroxide (IV).

In summary, the results obtained indicate the occurrence of an intermediate, proposed to be the flavin 4a-peroxyhemiacetal (III), which is formed in the reaction of the flavin 4ahydroperoxide (II) with aldehyde and precedes the formation of the excited state (IV*).

\section{REFERENCES}

Abu-Soud, H., Mullins, L. S., Baldwin, T. O., \& Raushel, F. M. (1992) Biochemistry 31, 3807-3813.

Baldwin, T. O., \& Ziegler, M. (1991) in Chemistry and Biochemistry of Flavoenzymes (Muller, F., Ed.) Vol. III, pp 467-530, CRC Press, Boca Raton, FL.

Eberhard, A., \& Hastings, J. W. (1972) Biochem. Res. Commun. 47, 348-353.

Gunsalus-Miguel, A., Meighen, E. A., Nicoli, M. Z., Nealson, K. H., \& Hastings, J. W. (1972) J. Biol. Chem. 247, 398-404.

Hastings, J. W. (1983) J. Mol. Evol. 19, 309-321.

Hastings, J. W., \& Balny, C. (1975) J. Biol. Chem. 250, 72887293.

Hastings, J. W., Weber, K., Friedland, J., Eberhard, A., Mitchell, G. W., \& Gunsalus, A. (1969) Biochemistry 8, 4681-4689.

Hastings, J. W., Balny, C., LePeuch, C., \& Douzou, P. (1973) Proc. Natl. Acad. Sci. U.S.A. 70, 3468-3472.

Hastings, J. W., Potrikus, C. J., Gupta, S., Kurfürst, M., \& Makemson, J. C. (1985) Adv. Microbiol. Physiol. 26, 235291.

Holzman, T. F., \& Baldwin, T. O. (1982) Biochemistry 21,61946201.

Kurfürst, M., Ghisla, S. \& Hastings, J. W. (1984) Proc. Natl. Acad. Sci. U.S.A. 81, 2990-2994.

Kurfürst, M., Macheroux, P., Ghisla, S., \& Hastings, J. W. (1987) Biochem. Biophys. Acta 924, 104-110.

Matheson, I. B. C., \& Lee, J. (1983) Photochem. Photobiol. 38 , 231-240.

Nealson, K. H. (1978) Methods Enzymol. 57, 153-166.

Presswood, R. P., \& Hastings, J. W. (1979) Photochem. Photobiol. 30, 93-99.

Seebach, D., Erickson, B., \& Singh, G. (1966) J. Org. Chem. 31, 4303-4304.

Suzuki, K., Kaidoh, T., Katagiri, M., \& Tsuchiza, T. (1983) Biochim. Biophys. Acta 722, 297-301.

Ulitzur, S., \& Hastings, J. W. (1978) Proc. Natl. Acad. Sci. U.S.A. 75, 266-269.

Vervoort, J., Müller, F., Lee, J., van den Berg, W. A. M., \& Moonen, C. T. W. (1986) Biochemistry 25, 8062-8067. 